\title{
Formigas de solo e de bromélias em uma área de Mata Atlântica, Ilha de Santa Catarina, sul do Brasil: Levantamento de espécies e novos registros
}

\author{
Félix Baumgarten Rosumek ${ }^{1 *}$ \\ Mônica Antunes Ulysséa ${ }^{2}$ \\ Benedito Cortês Lopes ${ }^{3}$ \\ Josefina Steiner ${ }^{2}$ \\ Anne Zillikens ${ }^{4}$

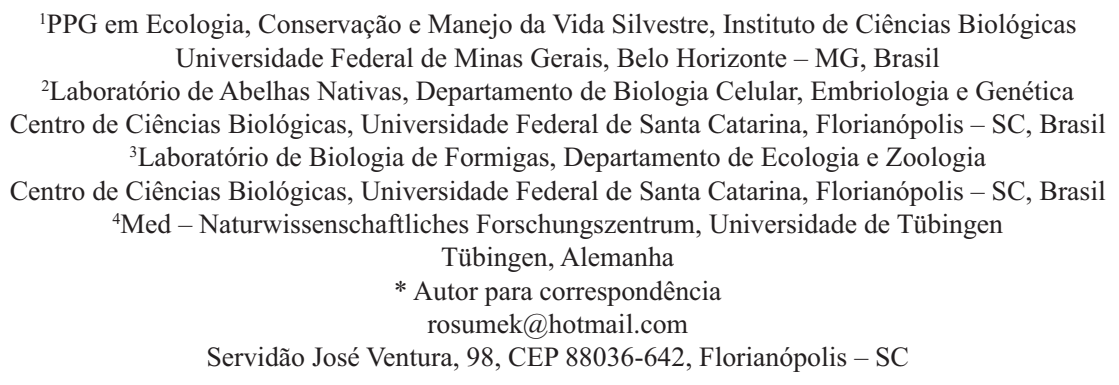

Submetido em 03/07/2008

Aceito para publicação em 05/08/2008

\section{Resumo}

A mirmecofauna em uma área de Mata Atlântica da Ilha de Santa Catarina foi inventariada utilizando métodos diversos para formigas de solo e de bromélias. Foram feitas amostragens mensais entre março de 2002 e agosto de 2004, utilizando o extrator de Winkler, armadilhas de interceptação e queda, além de coleta de bromélias. Foram encontradas 124 espécies, divididas em nove subfamílias e 33 gêneros. Os gêneros com maior riqueza foram Pheidole, Solenopsis, Crematogaster, Acromyrmex e Camponotus. Encontrou-se mais espécies no solo que nas bromélias, com $8 \%$ do total ocorrendo nos dois hábitats. Foram obtidos 11 registros novos para o estado de Santa Catarina e 20 para a região da Grande Florianópolis. Tais resultados estão relacionados com o uso de metodologias novas para a região, e mostram que a fauna de formigas desta ainda precisa ser mais bem estudada.

Unitermos: Formicidae, Mata Atlântica, formigas de solo, formigas de vegetação, bromélias

\section{Abstract}

Soil ants and bromeliad-nesting ants in an Atlantic Forest area, Santa Catarina Island, southern Brazil: Species inventory and new records. The myrmecofauna in an Atlantic Forest area was studied using various methods for soil ants and bromeliad-nesting ants. Monthly collections were performed between March 2002 and August 2004, using a Winkler extractor, pitfall traps and bromeliad individuals. One hundred and 
twenty-four species from nine subfamilies and 33 genera were found. The richest genera were Pheidole, Solenopsis, Crematogaster, Acromyrmex and Camponotus. More species were found in soil than in bromeliads, with $8 \%$ occurring in both habitats. Twelve species are new records for Santa Catarina State and 19 are new for the greater Florianópolis region. These results correlate with the use of new methodologies for the region, showing that the ant fauna of Santa Catarina Island still needs to be better studied.

Key words: Formicidae, Atlantic forest, soil ants, plant ants, bromeliads

\section{Introdução}

A Mata Atlântica é um dos ecossistemas mais ricos do planeta, considerado um dos 25 hotspots mundiais de biodiversidade. É a segunda maior floresta pluvial do continente americano, cobrindo originalmente 1,5 milhões de $\mathrm{km}^{2}, 92 \%$ desta área localizada no Brasil (Fundação SOS Mata Atlântica e INPE, 2001; GalindoLeal e Câmara, 2003). O ecossistema também é um dos mais ameaçados do mundo, já tendo perdido $93 \%$ de sua área (Morellato e Haddad, 2000). Mesmo assim, ainda abriga uma imensa biodiversidade e grande número de espécies endêmicas (Myers et al., 2000). Inventários de fauna são trabalhos fundamentais para a compreensão dos ecossistemas, particularmente daqueles sob forte influência antrópica, servindo de subsídios para estudos biogeográficos, ecológicos e trabalhos de conservação (Castri et al., 1992).

A família Formicidae (Classe Hexapoda, Ordem Hymenoptera) é considerada um dos grupos de invertebrados mais importantes e abundantes em grande parte dos ecossistemas terrestres. Esta importância é demonstrada por vários atributos: alta diversidade; predominância numérica e de biomassa em quase todos os hábitats do mundo; importantes funções nos ecossistemas, incluindo interações com organismos de todos os níveis tróficos (Alonso e Agosti, 2000). Alguns autores (Majer, 1983; Silva e Brandão, 1999) citam o fato de o estudo da riqueza de espécies de formigas ser útil para a avaliação da biodiversidade de invertebrados em geral.

A maioria das espécies de Formicidae está associada ao solo e à serapilheira (Wall e Moore, 1999; Pereira, 2006), mas interações variadas entre formigas e plantas são evolutivamente antigas e muito comuns (Delabie et al., 2003). Entre as diversas famílias de plantas relacionadas com formigas, podemos incluir a família Bromeliaceae (Blüthgen et al., 2000).
As bromélias (Classe Liliopsida, Ordem Bromeliales) ocorrem principalmente nas Américas, com uma espécie apenas na África (Reitz, 1983). As plantas na maior parte são epífitas e apresentam raízes apenas para auxiliar na fixação, mas também existem exemplares terrícolas (Joly, 1976; Reitz, 1983). O grupo tem grande importância entre as famílias vegetais que têm associações com a fauna, pois muitas das suas espécies provêm abrigos contra predadores, seca, vento e chuvas (Benzing, 2000). Sua disposição foliar tipo roseta permite a retenção de água nas bainhas e na região central da planta - dita copo, vaso ou tanque (Leme, 1984 apud Oliveira e Rocha, 1997). Por acumularem água e nutrientes em sua roseta, as bromélias constituem um microhábitat para inúmeras espécies de animais e plantas (Picado, 1913; Laessle, 1961; Frank e Lounibos, 1983; Reitz, 1983; Dejean e Olmsted, 1997; Mestre et al., 2001).

Observações preliminares sobre a biologia de formigas, descrevem que Formicidae e Bromeliaceae se ajustam em muitos tipos de interações (Benzing, 2000). A planta pode oferecer recursos alimentares, por exemplo, através de nectários extra-florais (Vesprini et al., 2003). A bromélia pode servir de ninho e conter em seu interior ovos, larvas, pupas e indivíduos reprodutivos alados de formigas (Wittman, 2000). Já as formigas podem beneficiar as bromélias através da proteção contra herbivoria, por dispersão de sementes e por alimentação com produtos abandonados em suas cavidades, que podem ser absorvidos pela planta (Dejean e Olmsted, 1997; Vesprini et al., 2003).

O presente artigo reúne os resultados de diversas coletas com formigas de solo e bromélias na Ilha de Santa Catarina, realizadas como parte de projetos mais amplos para estudo de fauna de solo e bromélias (ver Agradecimentos). O objetivo principal foi identificar a mirmecofauna que ocorre em bromélias e no solo em um 
fragmento de Mata Atlântica na Ilha de Santa Catarina, através do uso de técnicas de coleta variadas.

\section{Material e Métodos}

\section{Área de estudo}

A Ilha de Santa Catarina possui $425 \mathrm{~km}^{2}$, tendo uma forma alongada no sentido Norte-Sul. É paralela ao continente e separa-se deste por um estreito canal que forma as baías Norte e Sul (Caruso, 1983). O clima da região é Mesotérmico Úmido (Cfa), segundo a classificação de Köeppen, sendo caracterizado por altas taxas de umidade, chuvas distribuídas uniformemente durante $\mathrm{o}$ ano e temperaturas médias abaixo de $18^{\circ} \mathrm{C}$ no mês mais frio e superior a $22^{\circ} \mathrm{C}$ no mês mais quente. A precipitação média anual é em torno de $1500 \mathrm{~mm}$ (Mendonça, 1988) e a temperatura média anual é de 20, $4^{\circ} \mathrm{C}$ (Caruso, 1983).

O trabalho de campo foi executado em dois locais: na Unidade de Conservação Ambiental Desterro (UCAD) e em um sítio particular no bairro Santo Antônio de Lisboa. A UCAD está situada nas encostas da região noroeste da Ilha, sendo administrada pela Universidade Federal de Santa Catarina (UFSC), com o apoio do governo do Estado. A área possui 491,5 ha, cerca de $1,1 \%$ do território da Ilha (CECCA, 1997), abrigando Mata Atlântica secundária em diversos estágios de regeneração, algumas áreas de cobertura herbácea, assim como áreas de floresta com estrutura primária conservada. A área do sítio localiza-se a aproximadamente $3 \mathrm{~km}$ da UCAD, ambas se localizando no mesmo complexo de fragmentos. A topografia é caracterizada por uma encosta coberta por Mata Atlântica secundária com solo terroso e rochoso, apresentando grande riqueza de bromélias terrícolas e rupícolas.

\section{Procedimentos de campo}

Para este trabalho, utilizaram-se três técnicas de coleta: extrator de Winkler, armadilhas de queda (ambos para fauna de solo), além de coleta de bromélias.

As coletas de serapilheira para o extrator de Winkler foram realizadas no sítio particular. Foram feitas doze coletas mensais, entre setembro de 2003 e setembro de 2004. Em cada coleta foi recolhida uma amostra de $1 \mathrm{~m}^{2}$ de serapilheira. O material foi processado por $48 \mathrm{~h}$ no extrator de Winkler. Uma descrição mais detalhada do método pode ser encontrada em Bestelmeyer et al. (2000).

As coletas com armadilhas de queda (pitfalls) foram realizadas na UCAD, em uma encosta com Mata Atlântica em avançado estágio de regeneração. Foram feitas doze coletas mensais, entre setembro de 2003 e agosto de 2004. Em cada coleta utilizaram-se 25 armadilhas, gerando um esforço amostral total de 300 armadilhas. Estas foram dispostas em um transecto de $125 \mathrm{~m}$ e espaçadas $5 \mathrm{~m}$ entre si. As armadilhas consistiam de copos plásticos de $500 \mathrm{ml}$ preenchidos com $250 \mathrm{ml}$ de formol $2 \%$, cobertas com um quadrado de madeira de $15 \mathrm{~cm}$ x $15 \mathrm{~cm}$, a $5 \mathrm{~cm}$ do solo, para evitar inundação por chuvas e queda de matéria orgânica. As armadilhas permaneceram abertas por uma semana em cada período de coleta.

Cinco espécies de bromélias foram selecionadas para este estudo, sendo coletadas aleatoriamente na UCAD em saídas mensais, entre março de 2002 e junho de 2004. As plantas foram retiradas do substrato com a ajuda de uma faca, individualmente acondicionadas em sacos plásticos, identificadas e levadas ao laboratório. Foi coletado um total de 73 indivíduos de Aechmea lindenii (E. Morren) Baker, 61 de Aechmea nudicaulis (Linnaeus) Grisebach, 61 de Canistrum lindenii (Regel) Mez, 99 de Nidularium innocentii Lemaire (terrícolas) e 39 de Vriesea vagans (L. B. Smith) L. B. Smith (arborícola, coletados em árvores entre 10 e 15m).

\section{Análises}

O material obtido de cada método foi triado em laboratório e conservado em álcool a $70 \%$. As formigas foram separadas em grupos morfológicos e um ou dois exemplares de cada grupo montado em triângulos de papel para posterior identificação. Esta, a nível genérico, foi feita utilizando a chave de Palácio e Fernández (2003). Para a identificação de espécies, foi utilizada a literatura disponível e a comparação com exemplares da coleção de formigas do Museu de Zoologia da Universidade de São Paulo (MZUSP). Os grupos que 
não puderam ser identificados ao nível específico foram mantidos em morfoespécies.

Nosso trabalho reúne dados sem uma padronização rigorosa, obtidos com diferentes técnicas de coletas, em diferentes áreas e períodos de tempo. Portanto, não aplicamos análises quantitativas comparativas entre as áreas e hábitats em questão, como freqüência, composição ou estimativas de riqueza de espécies. Entretanto, alguns padrões foram identificados e são comentados na Discussão.

As notas sobre novas ocorrências no Estado e na região da Grande Florianópolis seguem os critérios e divisão por regiões utilizada na síntese de Silva (1999) sobre estudos mirmecológicos em Santa Catarina. $\mathrm{O}$ autor reuniu os dados da literatura e conferiu os espécimes depositados na coleção do MZUSP para criar uma lista das espécies encontradas nas diversas regiões do Estado. O autor utilizou o símbolo "?" para mostrar que há um dado na literatura relatando a ocorrência da espécie, mas o exemplar não foi encontrado em coleções para conferência. Compararam-se as espécies identificadas no presente trabalho com essa lista e trabalhos publicados posteriormente no Estado (Lopes e Fowler, 2000; Silva e Silvestre, 2000 e 2004; Lutinski e Garcia, 2005; Schmidt et al., 2005). Com exceção de Lopes e Fowler (2000), não foram publicados novos levantamentos para a região da Grande Florianópolis desde a revisão do autor.

O material está depositado nas coleções dos Laboratórios de Abelhas Nativas e do Laboratório de Biologia de Formigas, ambos localizados no Centro de Ciências Biológicas da Universidade Federal de Santa Catarina.

\section{Resultados}

Foram encontradas 124 espécies de Formicidae (58 identificadas e 66 morfoespécies), distribuídas entre nove subfamílias e 33 gêneros. As subfamílias encontradas foram (sensu Bolton, 2003): Dolichoderinae (3 gên., 7 spp.), Ecitoninae (2 gên., 2 spp.), Ectatomminae (1 gên, 4 spp.), Formicinae (5 gên., 17 spp.), Heteroponerinae (1 gên., 3 spp.), Myrmicinae (15 gên., 75 spp.),
Ponerinae (4 gên., 14 spp.), Proceratiinae (1 gên, 1 sp.) e Pseudomyrmecinae (1 gên, 1 sp.). Os gêneros com maior número de espécies foram Pheidole (25 spp.), Solenopsis (11 spp.), Crematogaster (9 spp.), Acromyrmex (7 spp.) e Camponotus (7 spp.) (Tabela 1).

Foram encontradas mais espécies no solo ( 81 espécies, sendo 71 exclusivas) do que nas bromélias (53 espécies, sendo 43 exclusivas). Apenas 10 espécies ( $8 \%$ do total) foram encontradas tanto no solo como nas bromélias. Nidularium innocentii foi a bromélia que apresentou maior frequência de formigas (em $67 \%$ dos indivíduos), seguida de C. lindenii (64\%), A. lindenii (55\%), A. nudicaulis (51\%) e $V$. vagans (49\%) (Tabela 1$)$.

Foram obtidos 11 registros novos de espécies para o Estado de Santa Catarina e 20 para a região da Grande Florianópolis (que inclui a Ilha de Santa Catarina e adjacências continentais). Para três espécies havia apenas o registro na literatura, mas sem que houvesse exemplares disponíveis para conferência (simbolizadas por “?”). Heteroponera dentinodus já havia sido encontrada no Estado, porém procedente de localidade desconhecida (simbolizada por "D") (Tabela 2).

Das espécies registradas pela primeira vez no Estado, cinco foram encontradas no solo e quatro nas bromélias. Para registros na Grande Florianópolis, dez foram encontrados em solo e sete nas bromélias. Wasmannia affinis e $W$. auropunctata foram os únicos novos registros a serem encontrados tanto em solo quanto nas bromélias (Tabelas 1 e 2).

\section{Discussão}

Silva (1999) lista 342 espécies e 72 gêneros de Formicidae para o Estado de Santa Catarina. Para a região da Grande Florianópolis, eram registradas 47 espécies identificadas, oriundas de diversos trabalhos utilizando metodologias como coleta manual, iscas e funil de Berlese (Lopes e Leal, 1991; Leal e Lopes, 1992; Bonnet e Lopes, 1993; Matos et al., 1994, Lopes e Santos, 1995; Silva e Lopes, 1997). Comparativamente, o presente trabalho apresentou um número alto de espécies identificadas (58). $\mathrm{O}$ número total de espécies (124) também foi alto em comparação a outros trabalhos na Mata Atlântica da Ilha: 
TABELA 1: Formigas de solo e de bromélias em Mata Atlântica da Ilha de Santa Catarina. As colunas da direita referem-se ao método / bromélia onde as espécies de formigas foram amostradas. WK - extrator de Winkler; PF - armadilha de queda (pitfall); AL - Aechmea lindenii; AN - Aechmea nudicaulis; CL - Canistrum lindenii; NI - Nidularium innocentii; VV - Vriesea vagans.

\begin{tabular}{|c|c|c|c|c|c|c|c|c|c|c|c|c|c|c|c|}
\hline Es pécie & WK & PF & $\mathbf{A L}$ & $\mathbf{A N}$ & $\mathbf{C L}$ & NI & VV & Espécie & WK & PF & $\mathbf{A L}$ & $\mathbf{A N}$ & $\mathbf{C L}$ & NI & $\mathbf{V V}$ \\
\hline DOLICHODERINAE & & & & & & & & MYRMICINAE & & & & & & & \\
\hline Dolichoderus attelaboides & & & & $\mathrm{x}$ & & & & Oxyepoecus crassinodus & & $\mathrm{x}$ & & & & & \\
\hline Linepithema cf. humile & & $\mathrm{x}$ & & & & & & Pheidole sp.1 & $\mathrm{x}$ & & & & & & \\
\hline Linepithema iniquum & & & & & & $\mathrm{x}$ & & Pheidole sp. 2 & $\mathrm{x}$ & $\mathrm{x}$ & & & & & \\
\hline Linepithema micans & & & & $\mathrm{x}$ & & & & Pheidole sp. 3 & $\mathrm{x}$ & & & & & & \\
\hline Linepithema sp.1 & $\mathrm{x}$ & & & & & & & Pheidole sp.4 & $\mathrm{x}$ & & & & & & \\
\hline Tapinoma melanocephalum & & & & & & & $\mathrm{x}$ & Pheidole sp.5 & $\mathrm{x}$ & & & & & & \\
\hline Tapinoma sp. 1 & & & & & $\mathrm{x}$ & & & Pheidole sp.6 & $\mathrm{x}$ & & & & & & \\
\hline ECITONINAE & & & & & & & & Pheidole sp.7 & $\mathrm{x}$ & $\mathrm{x}$ & & & & & \\
\hline Eciton burchelli & & $\mathrm{x}$ & & & & & & Pheidole sp.8 & & $\mathrm{x}$ & & & & & \\
\hline Labidus coecus & & $\mathrm{x}$ & & & & & & Pheidole sp.9 & & $\mathrm{x}$ & & & & & \\
\hline $\begin{array}{r}\text { ECTATOMMINAE } \\
\end{array}$ & & & & & & & & \begin{tabular}{|l|} 
Pheidole sp.10 \\
\end{tabular} & & $\mathrm{x}$ & & & & & \\
\hline Gnamptogenys moelleri & & & $\mathrm{x}$ & & & & $\mathrm{x}$ & \begin{tabular}{|l|} 
Pheidole sp.11 \\
\end{tabular} & & $\mathrm{x}$ & & & & & \\
\hline Gnamptogenys rastrata & & $\mathrm{x}$ & & & & & & \begin{tabular}{|l|} 
Pheidole sp. 12 \\
\end{tabular} & & $\mathrm{x}$ & & & & & \\
\hline Gnamptogenys striatula & $\mathrm{x}$ & $\mathrm{x}$ & $\mathrm{x}$ & $\mathrm{x}$ & $\mathrm{x}$ & $\mathrm{x}$ & $\mathrm{x}$ & Pheidole sp. 13 & & $\mathrm{x}$ & & & & & \\
\hline Gnamptogenys sp.1 & & & & & $\mathrm{x}$ & & & Pheidole sp.14 & & $\mathrm{x}$ & & & & & \\
\hline FORMICINAE & & & & & & & & Pheidole sp.15 & & $\mathrm{x}$ & & & & & \\
\hline Acropyga goeldii & & $\mathrm{x}$ & & & & & & \begin{tabular}{|l|} 
Pheidole sp. 16 \\
\end{tabular} & & $\mathrm{x}$ & & & & & \\
\hline Brachymyrmex cf. admotus & & & & & & $\mathrm{x}$ & & \begin{tabular}{|l|} 
Pheidole sp.17 \\
\end{tabular} & & $\mathrm{x}$ & & & & & \\
\hline Brachymyrmex coactus & & & $\mathrm{x}$ & & $\mathrm{x}$ & & & Pheidole sp. 18 & & $\mathrm{x}$ & & & & & \\
\hline Brachymyrmex sp.1 & $\mathrm{x}$ & $\mathrm{x}$ & & & & & & Pheidole sp.19 & & $\mathrm{x}$ & & & & & \\
\hline Brachymyrmex sp. 2 & & $\mathrm{x}$ & & & & & & \begin{tabular}{|l|} 
Pheidole sp.20 \\
\end{tabular} & & & $\mathrm{x}$ & $\mathrm{x}$ & $\mathrm{x}$ & & \\
\hline Camponotus bonariensis & & & & $\mathrm{x}$ & & & & \begin{tabular}{|l|} 
Pheidole sp. 21 \\
\end{tabular} & & & $\mathrm{x}$ & & & $\mathrm{x}$ & $\mathrm{x}$ \\
\hline Camponotus cf. fastigatus & $\mathrm{x}$ & $\mathrm{x}$ & $\mathrm{x}$ & & & & & Pheidole sp.22 & & & $\mathrm{x}$ & $\mathrm{x}$ & & $\mathrm{x}$ & $\mathrm{x}$ \\
\hline Camponotus lespesii & & $\mathrm{x}$ & & & & & $\mathrm{x}$ & Pheidole sp. 23 & & & $\mathrm{x}$ & & & & \\
\hline Camponotus cf. melanoticus & & & & $\mathrm{x}$ & & & & \begin{tabular}{|l|} 
Pheidole sp.24 \\
\end{tabular} & & & $\mathrm{x}$ & & & & \\
\hline Camponotus cf. rufipes & & & $\mathrm{x}$ & $\mathrm{x}$ & & & & Pheidole sp. 25 & & & & & & $\mathrm{x}$ & \\
\hline Camponotus sericeiventris & & $\mathrm{x}$ & & & & & & Procryptocerus grupo peltatus & & & & & $\mathrm{x}$ & & \\
\hline Camponotus (Myrmobrachys) sp.1 & & & $\mathrm{x}$ & & & & & Pyramica denticulata & $\mathrm{x}$ & & & & & & \\
\hline Myrmelachista catharinae & & & & & $\mathrm{x}$ & $\mathrm{x}$ & & Monomorium sp.1 & & $\mathrm{x}$ & & & & & \\
\hline Paratrechina longicornis & $\mathrm{x}$ & $\mathrm{x}$ & & & & & & \begin{tabular}{|l|} 
Solenopsis sp.1 \\
\end{tabular} & $\mathrm{x}$ & $\mathrm{x}$ & & & & & \\
\hline Paratrechina sp.1 & $\mathrm{x}$ & & & & & & & Solenopsis sp.2 & $\mathrm{x}$ & & & & & & \\
\hline Paratrechina $\mathrm{sp} .2$ & & $\mathrm{x}$ & & & & & & Solenopsis sp.3 & $\mathrm{x}$ & $\mathrm{x}$ & & & & & \\
\hline Paratrechina sp. 3 & & $\mathrm{x}$ & & & & & & \begin{tabular}{|l|} 
Solenopsis sp.4 \\
\end{tabular} & $\mathrm{x}$ & & & & & & \\
\hline HETEROPONERINAE & & & & & & & & Solenopsis $\mathrm{sp} .5$ & $\mathrm{x}$ & & & & & & \\
\hline Heteroponera dentinodus & & $\mathrm{x}$ & & & $\mathrm{x}$ & $\mathrm{x}$ & & \begin{tabular}{|l|} 
Solenopsis sp.6 \\
\end{tabular} & $\mathrm{x}$ & $\mathrm{x}$ & & & & & \\
\hline Heteroponera inermis & & $\mathrm{x}$ & & & & & & \begin{tabular}{|l|} 
Solenopsis sp. 7 \\
\end{tabular} & $\mathrm{x}$ & & & & & & \\
\hline Heteroponera mayri & & & & & $\mathrm{x}$ & $\mathrm{x}$ & & \begin{tabular}{|l|} 
Solenopsis sp. 8 \\
\end{tabular} & $\mathrm{x}$ & $\mathrm{x}$ & & & & & \\
\hline MYRMICINAE & & & & & & & & Solenopsis sp.9 & $\mathrm{x}$ & $\mathrm{x}$ & & & & & \\
\hline Acromyrmex aspersus & $\mathrm{x}$ & $\mathrm{x}$ & & & & & & Solenopsis sp.10 & $\mathrm{x}$ & $\mathrm{x}$ & & & & & \\
\hline Acromyrmex hispidus falax & & & & & $\mathrm{x}$ & & & \begin{tabular}{|l} 
Solenopsis sp.11 \\
\end{tabular} & $\mathrm{x}$ & $\mathrm{x}$ & & & & & \\
\hline Acromyrmex hystrix & & & & & $\mathrm{x}$ & & & \begin{tabular}{|l|} 
Strumigenys eggersi \\
\end{tabular} & $\mathrm{x}$ & & & & & & \\
\hline Acromyrmex laticeps & $\mathrm{x}$ & & & & & & & Strumigenys elongata & $\mathrm{x}$ & & & & & & \\
\hline Acromyrmex niger & & & $\mathrm{x}$ & & & & & Strumigenys perparva & $\mathrm{x}$ & & & & & & \\
\hline Acromyrmex subterraneus & $\mathrm{x}$ & $\mathrm{x}$ & $\mathrm{x}$ & & & & & \begin{tabular}{|l|} 
Strumigenys sp. 1 \\
\end{tabular} & $\mathrm{x}$ & & & & & & \\
\hline Acromyrmex sp.1 & & $\mathrm{x}$ & & & & & & Strumigenys sp. 2 & $\mathrm{x}$ & & & & & & \\
\hline Apterostigma $\mathrm{sp} .1$ & & $\mathrm{x}$ & & & & & & Wasmannia affinis & $\mathrm{x}$ & $\mathrm{x}$ & & & $\mathrm{x}$ & $\mathrm{x}$ & \\
\hline Apterostigma sp.2 & & $\mathrm{x}$ & & & & & & Wasmannia auropunctata & $\mathrm{x}$ & $\mathrm{x}$ & & $\mathrm{x}$ & $\mathrm{x}$ & $\mathrm{x}$ & \\
\hline Apterostigma $\mathrm{sp} .3$ & & $\mathrm{x}$ & & & & & & PONERINAE & & & & & & & \\
\hline Carebara cf. bicarinatus & $\mathrm{x}$ & & & & & & & Hypoponera sp.1 & $\mathrm{x}$ & $\mathrm{x}$ & & & & & \\
\hline Crematogaster nigropilosa & & & & $\mathrm{x}$ & & & & Hypoponera sp.2 & & & $\mathrm{x}$ & & & $\mathrm{x}$ & \\
\hline Crematogaster sp. 1 & $\mathrm{x}$ & $\mathrm{x}$ & $\mathrm{x}$ & $\mathrm{x}$ & $\mathrm{x}$ & & $\mathrm{x}$ & Hypoponera sp.3 & & & & $\mathrm{x}$ & & $\mathrm{x}$ & \\
\hline Crematogaster sp. 2 & $\mathrm{x}$ & & $\mathrm{x}$ & $\mathrm{x}$ & & $\mathrm{x}$ & & Hypoponera sp.4 & & & $\mathrm{x}$ & & & & $\mathrm{x}$ \\
\hline Crematogaster sp. 3 & & & $\mathrm{x}$ & $\mathrm{x}$ & $\mathrm{x}$ & $\mathrm{x}$ & & Odontomachus chelifer & $\mathrm{x}$ & $\mathrm{x}$ & & & & & \\
\hline Crematogaster sp.4 & & & & $\mathrm{x}$ & & & & Odontomachus meinerti & $\mathrm{x}$ & $\mathrm{x}$ & & & & & \\
\hline Crematogaster sp.5 & & & $\mathrm{x}$ & $\mathrm{x}$ & & & & \begin{tabular}{|l|} 
Odontomachus $\mathrm{sp} .1$ \\
\end{tabular} & & & $\mathrm{x}$ & & & & \\
\hline Crematogaster sp. 6 & & & $\mathrm{x}$ & $\mathrm{x}$ & $\mathrm{x}$ & & $\mathrm{x}$ & Pachycondyla bucki & & $\mathrm{x}$ & & & & & \\
\hline Crematogaster sp.7 & & & $\mathrm{x}$ & $\mathrm{x}$ & & & & Pachycondyla crenata & & & $\mathrm{x}$ & & $\mathrm{x}$ & & $\mathrm{x}$ \\
\hline Crematogaster $\mathrm{sp} .8$ & & & & & & & $\mathrm{x}$ & Pachycondyla inversa & & & & $\mathrm{x}$ & & & \\
\hline Cyphomyrmex auritus & & & $\mathrm{x}$ & & $\mathrm{x}$ & $\mathrm{x}$ & & Pachycondyla marginata & & $\mathrm{x}$ & & & & & \\
\hline Cyphomyrmex peltatus & & & & $\mathrm{x}$ & & $\mathrm{x}$ & & Pachycondyla striata & $\mathrm{x}$ & $\mathrm{x}$ & & & & $\mathrm{x}$ & \\
\hline Cyphomyrmex rimosus & $\mathrm{x}$ & $\mathrm{x}$ & & & & & & Pachycondyla villosa & & & & $\mathrm{x}$ & & & \\
\hline Cyphomyrmex sp.1 & & & & & & $\mathrm{x}$ & & Thaumatomyrmex mutilatus & $\mathrm{x}$ & & & & & & \\
\hline Hylomyrma reitteri & $\mathrm{x}$ & $\mathrm{x}$ & & & & & & PROCERATIINAE & & & & & & & \\
\hline Octostruma jheringi & & $\mathrm{x}$ & & & & & & Discothyrea neotropica & $\mathrm{x}$ & $\mathrm{x}$ & & & & & \\
\hline Octostruma petiolata & $\mathrm{x}$ & $\mathrm{x}$ & & & & & & PSEUDOMYRMECINAE & & & & & & & \\
\hline Octostruma rugifera & $\mathrm{x}$ & & & & & - & & Pseudomyrmex phyllophilus & & & & & $\mathrm{x}$ & & \\
\hline
\end{tabular}


TABELA 2: Novos registros de formigas para o estado de Santa Catarina e a região da Grande Florianópolis. GF - primeiro registro na região da Grande Florianópolis; SC primeiro registro em Santa Catarina; D - exemplar existente em coleções, mas com localidade desconhecida; ? - dado na literatura relatando a ocorrência da espécie em Santa Catarina, mas o exemplar não foi encontrado em coleções para conferência (segundo Silva, 1999).

\begin{tabular}{l|c}
\multicolumn{1}{c|}{ Espécie } & Registro \\
\hline Dolichoderus attelaboides & GF \\
\hline Linepithema cf. humile & GF \\
\hline Linepithema iniquum & SC \\
\hline Linepithema micans & SC \\
\hline Tapinoma melanocephalum & GF \\
\hline Labidus coecus & GF \\
\hline Gnamptogenys rastrata & SC \\
\hline Acropyga goeldii & SC \\
\hline Brachymyrmex cf. admotus & GF \\
\hline Bracymyrmex coactus & GF \\
\hline Camponotus bonariensis & $?$ \\
\hline Camponotus lespesii & GF \\
\hline Crematogaster nigropilosa & GF \\
\hline Myrmelachista catharinae & $?$ \\
\hline Paratrechina longicornis & D \\
\hline Heteroponera dentinodus & GF \\
\hline Heteroponera inermis & GF \\
\hline Heteroponera mayri & GF \\
\hline Acromyrmex aspersus & SC \\
\hline Acromyrmex hystrix & SC \\
\hline Carebara cf. bicarinatus & GF \\
\hline Cyphomyrmex auritus & GF \\
\hline Cyphomyrmex peltatus & GF \\
\hline Cyphomyrmex rimosus & SC \\
\hline Octostruma jheringi & GF \\
\hline Octostruma rugifera & GF \\
\hline Oxyepoecus crassinodus & SC \\
\hline Strumigenys perparva & SC \\
\hline Wasmannia affinis & GF \\
\hline Wasmannia auropunctata & GF \\
\hline Pachycondyla bucki & SC \\
\hline Pachycoldyla inversa & SC \\
\hline Pachycondyla marginata & GF \\
\hline Thaumatomyrmex mutilatus & GF \\
\hline Discothyrea neotropica & \\
\hline & \\
\hline
\end{tabular}

Leal e Lopes (1992) registraram 63 espécies, enquanto Silva e Lopes (1997) registraram 74 espécies diurnas. Tais resultados são relacionados ao uso de duas técnicas eficientes para coleta de formigas de solo (extrator de Winkler e armadilhas de queda) e da coleta em bromélias, todos ainda não empregadas na região. Mesmo com coletas anteriores de formigas em serapilheira, houve mais registros novos no solo que nas bromélias, indicando a necessidade de métodos variados para amostragens precisas. Como apontado por Bestelmeyer et al. (2000), cada método apresenta diferentes eficiências para capturar formigas de hábitos distintos.

A maior riqueza de formigas no solo do que nas plantas é esperada, visto que a maioria das espécies da família está associada com o solo e a serapilheira (Wall e Moore, 1999; Pereira, 2006). A pequena sobreposição de espécies entre solo e bromélias indica uma especialização da maioria das formigas a um hábitat em especial. Pheidole e Solenopsis representaram 29\% das espécies encontradas (36\% das encontradas no solo). Estes dois gêneros representam, juntos, cerca de $22 \%$ das espécies de Formicidae do mundo (Schütte et al., 2007). Solenopsis foi encontrado apenas no solo, enquanto apenas seis das 25 espécies de Pheidole foram encontradas em bromélias, sendo que nenhuma espécie ocorreu nos dois hábitats. Pheidole é um gênero hiperdiverso, predominante no solo de quase todos os ecossistemas florestais da região neotropical, mas apresentando várias espécies especializadas em hábitats arborícolas (Wilson, 2003).

Por outro lado, Crematogaster é um gênero predominantemente associado à vegetação, mas com poucas espécies de solo (Wilson, 1976; Longino, 2003), o que explica a distribuição das espécies encontradas: sete espécies exclusivas de bromélias e duas encontradas nos dois hábitats. Segundo Brown (1973), Crematogaster tem ampla distribuição mundial, sendo encontrado em todos os ambientes, e possui hábito alimentar onívoro. As espécies deste gênero são também oportunistas em termos de locais de nidificação, freqüentemente associadas a diversos tipos de plantas, como as bromélias que, ao acumularem água e nutrientes na sua roseta foliar, constituem um microhábitat para inúmeras espécies de insetos, além das formigas. 
Foi obtida uma grande quantidade de novos registros para o estado e a região. Nosso trabalho registrou um total de 31 novas espécies para a Grande Florianópolis, um número comparativamente alto às 47 espécies conhecidas anteriormente. Embora sem dados publicados na literatura, Octostruma petiolata já foi registrada na região da Grande Florianópolis (Dietz, 2004). Pachycondyla bucki foi registrada previamente em Santa Catarina (município de São Bento do Sul), mas não na região da Grande Florianópolis (Silva, 2004). Outra vez, podemos atribuir estes números à combinação de técnicas de coleta utilizadas. Muitos dos novos registros são de formigas pequenas e crípticas (Carebara cf. bicarinata, Discothyrea neotropica, Octostruma rugifera, Strumigenys perparva), amostradas através do extrator de Winkler e dificilmente coletadas através de métodos menos eficientes para a fauna hipogéica. Neste trabalho constam dados de 12 unidades amostrais de $1 \mathrm{~m}^{2}$ de serapilheira para o Winkler. O protocolo de coleta para formigas de serapilheira sugerido por Agosti e Alonso (2000 e 2003) recomenda que cada unidade amostral seja composta por 20 amostras de $1 \mathrm{~m}^{2}$ de serapilheira. O nosso esforço amostral para coletas com o extrator de Winkler ainda é pequeno, se comparado a outros trabalhos.

Além dos métodos, a disponibilidade de chaves recentes também ajudou a ampliar o número de novos registros. As espécies de Linepithema e Wasmannia foram identificadas com recentes revisões de Wild (2007) e Longino e Fernández (2007), respectivamente.

É importante notar que alguns dos novos registros referem-se a formigas muito comuns e previamente conhecidas, como Tapinoma melanocephalum (primeiro registro publicado para o Estado), Cyphomyrmex rimosus e Wasmannia auropunctata (primeiros registros publicados para a região). Isto reflete uma carência tanto de levantamentos faunísticos como de publicação de resultados na literatura especializada.

Outro problema, já notado por Silva (1999), é a dificuldade de identificação de vários dos gêneros com maior riqueza neste trabalho. Pheidole, Solenopsis e Crematogaster estão entre os dez gêneros com maior riqueza de espécies de formigas no mundo (Bolton, 1995). Entretanto, não existem chaves funcionais para sua identificação, assim como para diversos outros gêneros encontrados. É bem possível que, dentre as 66 morfoespécies listadas aqui, haja diversas espécies ainda não registradas para a região.

Devido ao alto número de espécies e novos registros encontrados, em comparação aos trabalhos anteriores, concluímos que as faunas mirmecológicas da Ilha e do estado de Santa Catarina ainda carecem de estudos, em especial no tocante às formigas arborícolas e terrícolas de formações vegetais complexas como a Mata Atlântica. Salientamos a necessidade de técnicas variadas para a coleta de espécies de hábitos distintos. Novos estudos, com métodos elaborados e em uma maior diversidade de ambientes, ajudarão a formar um painel preciso da fauna de formigas da região.

\section{Agradecimentos}

Ao pessoal do Laboratório de Abelhas Nativas, pela coleta e triagem inicial do material. Ao Dr. Rogério Rosa da Silva, Dr. Nicolas Lavor de Albuquerque e especialmente Msc. Rodrigo dos Santos Machado Feitosa, pelo auxílio na identificação de espécies no MZUSP. Este estudo faz parte dos projetos "Importância das bromélias para a manutenção da biodiversidade da fauna associada na Mata Atlântica" $(\mathrm{CNPq}$, processo 690143/01-O) e "Internal dynamics of rain forests: specificity of animal - plant interactions" (BMBF, Alemanha, 01LB0205) dentro do programa Brasil Alemanha "Mata Atlântica".

\section{Referências}

Agosti, D.; Alonso, L. E. 2000. The ALL Protocol. In: Agosti, D.; Majer, J. D.; Alonso, L. E. \& Schultz, T. R. (Eds). Ants: Standard methods for measuring and monitoring biodiversity. Smithsonian Institution Press, Washington, USA, p.204-206.

Agosti, D.; Alonso, L. E. 2003. El protocolo ALL: Un estándar para la colección de hormigas del suelo. In: Agosti, D.; Majer, J. D.; Alonso, L. E. \& Schultz, T. R. (Eds). Ants: Standard methods for measuring and monitoring biodiversity. Smithsonian Institution Press, Washington, USA, p.415-418.

Alonso, L. E.; Agosti, D. 2000. Biodiversity studies, monitoring and ants: An overview. In: Agosti, D.; Majer, J. D.; Alonso, L. E. \& Schultz, T. R. (Eds). Ants: Standard methods for measuring and monitoring biodiversity. Smithsonian Institution Press, Washington, USA, p.1-8. 
Benzing, D. H. 2000. Ants and bromeliads. In: Benzing, D. H. (Ed.). Bromeliaceae: Profile of an adaptive radiation. Harvard University Press, Cambridge, USA, p.421-435.

Bestelmeyer, B. T.; Agosti, D.; Alonso, L. E.; Brandão, C. R. F.; Brown, W. J.; Delabie, J. H. C.; Silvestre, R. 2000. Field techniques for the study of ground-dwelling ants. In: Agosti, D.; Majer, J. D.; Alonso, L. E. \& Schultz, T. R. (Eds). Ants: Standard methods for measuring and monitoring biodiversity. Smithsonian Institution Press, Washington, USA, p.122-144.

Blüthgen, N.; Verhaagh, M.; Goitía, W. 2000. Ant nests in tank bromeliads - An example of non-specific interaction. Insectes Sociaux, 47: 313-316.

Bolton, B. 1995. A taxonomic and zoogeographical census of the extant ant taxa (Hymenoptera: Formicidae). Journal of Natural History, 29: 1037-1056.

Bolton, B. 2003. Synopsis and classification of Formicidae. Memoirs of the American Entomological Institute, 71: 1-370.

Bonnet, A.; Lopes, B. C. 1993. Formigas de dunas e restingas da praia da Joaquina, Ilha de Santa Catarina, SC (Insecta: Hymenoptera). Biotemas, 6 (1): 107-114.

Brown, W. L. 1973. A comparison of the Hylean and Congo-West African rain forest ant faunas. In: Meggers, B. J.; Ayensu, E. S. \& Duckworth, S. W. O. (Eds). Tropical forest ecosystems in Africa and South America: A comparative review. Smithsonian Institution Press, Washington, USA, p.161-185.

Caruso, M. M. L. 1983. O desmatamento da Ilha de Santa Catarina de 1500 aos dias atuais. EDUFSC, Florianópolis, Brasil, 158pp.

Castri, F.; Vernhes, J. R.; Younès, T. 1992. Inventorying and monitoring biodiversity: A proposal for an international network. Biology International, Special Issue, 27: 1-29.

CECCA - Centro de Estudos Cultura e Cidadania. 1997. Unidades de conservação e áreas protegidas da Ilha de Santa Catarina: Caracterização e legislação. Insular, Florianópolis, Brasil, 160pp.

Dejean, A.; Olmsted, I. 1997. Ecological studies on Aechmea bracteata (Swartz) (Bromeliaceae). Journal of Natural History, 31: 1313-1334.

Delabie, J. H. C.; Osprina, M.; Zabala, G. 2003. Relaciones entre hormigas y plantas: Una introducción. In: Fernández, F. (Ed.). Introducción a las hormigas de la región Neotropical. Instituto de Investigación de Recursos Biológicos Alexander Von Humboldt, Bogotá, Colômbia, p.167-180.

Dietz, B. H. 2004. Revisão da tribo Basicerotini (Hymenoptera : Formicidae). Tese de Doutorado, Universidade de São Paulo, Brasil, 245pp.

Frank, J. H.; Lounibos, L. P. 1983. Phytotelmata: Terrestrial plants as host for aquatic insect communities. Plexus, Medford, New Jersey, USA, 293pp.

Fundação SOS Mata Atlântica; INPE (Instituto Nacional de Pesquisas Espaciais). 2001. Atlas dos remanescentes florestais da Mata Atlântica e ecossistemas associados no período de 1995-2000. Fundação SOS Mata Atlântica e INPE, São Paulo, Brasil, 43pp.

Galindo-Leal, C.; Câmara, I. G. 2003. Atlantic forest hotspots status: An overview. In: Galindo-Leal, C. \& Câmara, I. G. (Eds). The Atlantic Forest of South America: Biodiversity status, threats, and outlook. Center for Applied Biodiversity Science and Island Press, Washington, USA, p.3-11.

Joly, A. B. 1976. Botânica: Introdução à taxonomia vegetal. $3^{\text {a }}$ ed. Editora Nacional, São Paulo, Brasil, 777pp.

Laessle, A. M. 1961. A micro-limnological study of Jamaican bromeliads. Ecology, 42: 499-517.

Leal, I. R.; Lopes, B. C. 1992. Estrutura das comunidades de formigas (Hymenoptera: Formicidae) de solo e vegetação no Morro da Lagoa da Conceição, Ilha de Santa Catarina, SC. Biotemas, 5 (1): 107-122.

Longino, J. T. 2003. The Crematogaster (Hymenoptera, Formicidae, Myrmicinae) of Costa Rica. Zootaxa, 151: 1-150.

Longino, J. T.; Fernández, F. 2007. Taxonomic review of the genus Wasmannia. In: Snelling, R. R.; Fisher, B. L. \& Ward, P. S. (Eds). Advances in ant systematics (Hymenoptera: Formicidae): Homage to E. O. Wilson - $\mathbf{5 0}$ years of contributions. American Entomological Institute, Gainesville, USA, p.271-289.

Lopes, B. C.; Fowler, H. G. 2000. Fungus-growing ants (Hymenoptera: Formicidae) on Santa Catarina Island, Brazil: Patterns of occurrence. Revista de Biología Tropical, 48 (2/3): 643-646.

Lopes, B. C.; Leal, I. 1991. Levantamento preliminar de formigar (Hymenoptera: Formicidae) de solo e vegetação em um trecho de mata atlântica, Morro da Lagoa da Conceição, Ilha de Santa Catarina, S.C. Biotemas, 4 (2): 51-59.

Lopes, B. C.; Santos, R. A. 1995. Formigas de manguezal: Espécies envolvidas e utilização das plantas. Anais do XII Encontro de Mirmecologia, São Leopoldo, Brasil, p.82.

Lutinski, J. A.; Garcia, F. R. M. 2005. Análise faunística de Formicidae (Hymenoptera: Apocrita) em ecossistema degradado no município de Chapecó, Santa Catarina. Biotemas, 18 (2): 73-86.

Majer, J. D. 1983. Ants: Bio-indicators of minesite rehabilitation, land use, and land conservation. Environmental Management, 7 (4): 375-383.

Matos, J. Z.; Yamanaka, C. N.; Castellani, T. T.; Lopes, B. C. 1994. Comparação da fauna de formigas de solo em áreas de plantio de Pinus eliottii, com diferentes graus de complexidade (Florianópolis, SC). Biotemas, 11 (1): 157-164.

Mendonça, M. 1988. Estudo preliminar de geomorfologia costeira na Ilha de Santa Catarina: Daniela e Ponta das Canas, mun. Florianópolis - SC. Geosul, 3 (5): 52-74.

Mestre, L. A. M.; Aranha, J. M. R.; Esper, M. L. 2001. Macroinvertebrate fauna associated to the bromeliad Vriesea inflata of Atlantic Forest (Parana state, southern Brazil). Brazilian Archives of Biology and Technology, 44: 89-94.

Morellato, L. P. C.; Haddad, C. F. B. 2000. Introduction: The Brazilian Atlantic Forest. Biotropica, 32 (4b): 786-792.

Myers, N.; Mittermeier, R. A.; Mittermeier, C. G.; Fonseca, G. A. B.; Kent, J. 2000. Biodiversity hotspots for conservation priorities. Nature, 403: 853-845.

Oliveira, M. G. N.; Rocha, C. F. D. 1997. O efeito da complexidade da bromélia-tanque Neoregelia cruenta (R. Graham) L. B. Smith sobre a comunidade animal associada. Bromélia, 4: 13-21.

Palácio, E. E.; Fernández, F. 2003. Claves para las subfamílias e géneros. In: Fernández, F. (Ed.). Introducción a las hormigas de la región Neotropical. Instituto de Investigación de Recursos Biológicos Alexander Von Humboldt, Bogotá, Colômbia, p.233-260. 
Pereira, A. 2006. A vida entre folhas secas. Pesquisa FAPESP, 120: 48-51.

Picado, C. 1913. Les broméliacées épiphytes considérées comme milieu biologique. Bulletin Scientifique France et Belgique, 5: 215-360.

Reitz, R. 1983. Bromeliáceas e a malária - Bromélia endêmica. Herbário Barbosa Rodrigues, Itajaí, Brasil, 808pp.

Schmidt, K.; Corbetta, R.; Camargo, A. J. A. 2005. Formigas (Hymenoptera: Formicidae) da Ilha João da Cunha, SC: Composição e diversidade. Biotemas, 18 (1): 57-71

Schütte, M. S.; Queiroz, J. M.; Mayhé-Nunes, A. J.; Pereira, M. P. S. 2007. Inventário estruturado de formigas (Hymenoptera, Formicidae) em floresta ombrófila de encosta na ilha da Marambaia, RJ. Iheringia, 97 (1): 103-110.

Silva, R. R. 2004. Estrutura de guildas de formigas (Hymenoptera: Formicidae) de serapilheira em quatro áreas de Floresta Atlântica do sul e sudeste do Brasil. Tese de Doutorado, Universidade de São Paulo, Brasil, 76pp.

Silva, R. R. 1999. Formigas (Hymenoptera: Formicidae) do oeste de Santa Catarina: Histórico de coletas e lista atualizada das espécies do Estado de Santa Catarina. Biotemas, 12 (2): 75-100.

Silva, R. R.; Brandão C. R. F. 1999. Formigas (Hymenoptera: Formicidae) como indicadores da qualidade ambiental e da biodiversidade de outros invertebrados terrestres. Biotemas, 12 (2): 55-73.

Silva, R. R.; Lopes, B. C. 1997. Ants (Hymenoptera: Formicidae) from Atlantic rainforest at Santa Catarina Island, Brazil: two years of sampling. Revista de Biología Tropical, 45 (4): 1641-1648.
Silva, R. R.; Silvestre, R. 2000. Diversidade de formigas (Hymenoptera: Formicidae) em Seara, oeste de Santa Catarina. Biotemas, 13 (2): 85-105.

Silva, R. R.; Silvestre, R. 2004. Riqueza da fauna de formigas (Hymenoptera: Formicidae) que habita as camadas superficiais do solo em Seara, Santa Catarina. Papéis Avulsos de Zoologia, 44 (1): $1-11$.

Vesprini, J. L.; Galetto, L.; Bernardello, G. 2003. The beneficial effect of ants on the reproductive success of Dyckia floribunda (Bromeliaceae), an extrafloral nectary plant. Canadian Journal of Botany, 81: 24-27.

Wall, D. H.; Moore, J. C. 1999. Interactions underground. BioScience, 49: 109-107.

Wild, A. L. 2007. Taxonomic revision of the ant genus Linepithema (Hymenoptera: Formicidae). University of California Publications in Entomology, 126: 1-151.

Wilson, E. O. 1976. Which are the most prevalent ant genera? Studia Entomologica, 19 (1-4): 187-200.

Wilson, E. O. 2003. Pheidole in the New World: A dominant, hyperdiverse ant genus. Harvard University Press, Cambridge, USA, $921 \mathrm{pp}$.

Wittman, P. K. 2000. The animal community associated with canopy bromeliads of the lowland Peruvian Amazon rain forest. Selbyana, 21: $48-51$. 